Article

\title{
Synthesis and In Vitro Antiproliferative Activity of 11-Substituted Neocryptolepines with a Branched $\omega$-Aminoalkylamino Chain
}

\author{
Elkhabiry Shaban ${ }^{1}$, Marta Świtalska ${ }^{2}$, Li Wang 1,3, Ning Wang 1,4 , Fan Xiu ${ }^{3}$, \\ Ikuya Hayashi ${ }^{1}$, Tran Anh Ngoc ${ }^{1}$, Sachie Nagae ${ }^{1}$, Samah El-Ghlban ${ }^{1}$, Shiho Shimoda ${ }^{1}$, \\ Ahmed Abdel Aleem El Gokha ${ }^{5}$, Ibrahim El Tantawy El Sayed 1,5,*, Joanna Wietrzyk ${ }^{2, *}$ (D) and \\ Tsutomu Inokuchi ${ }^{1, *}$ \\ 1 Division of Chemistry and Biotechnology, Graduate School of Natural Science and Technology, \\ Okayama University, 3-1-1 Tsushima-naka, Kita-ku, Okayama 700-8530, Japan; \\ shaban_nrc@yahoo.com (E.S.); liwang_512@163.com (L.W.); wn12171982@sina.com (N.W.); \\ en20753@s.okayama-u.ac.jp (I.H.); trananhngoc90@gmail.com (T.A.N.); kunio.hearn@gmail.com (S.N.); \\ S_elghlban@yahoo.com (S.E.-G.); n.n.n.ism.2@gmail.com (S.S.) \\ 2 Hirszfeld Institute of Immunology and Experimental Therapy, Polish Academy of Sciences, 12, \\ R. Weigla Street, 53-114 Wroclaw, Poland; switalska@iitd.pan.wroc.pl \\ 3 Department of Medicinal Chemistry, School of Pharmacy, Southwest Medical University, \\ Luzhou 646000, China; 18090755675@163.com \\ 4 Department of Chemistry and Materials Engineering, Yingkou Institute of Technology, \\ Yingkou 115014, China \\ 5 Department of Chemistry, Faculty of Science, Menoufia University, Shebin El Koom 32511, Egypt; \\ aelgokha@yahoo.com \\ * Correspondence: ibrahimtantawy@yahoo.co.uk (I.E.T.E.S.); wietrzyk@iitd.pan.wroc.pl (J.W.); \\ inokuchi@cc.okayama-u.ac.jp (T.I.); Tel.: +2-048-2235689 (I.E.T.E.S.); +48-71-337-2171 (J.W.); \\ +81-86-294-5045 (T.I.)
}

Received: 14 October 2017; Accepted: 10 November 2017; Published: 12 November 2017

\begin{abstract}
Neocryptolepine, which is a kind of tetracyclic indoloquinoline alkaloid, exhibits the inhibition of topoisomerase II and shows antiproliferative activity. The present study describes the synthesis and antiproliferative evaluation of several neocryptolepine analogues carrying a branched, functionalized dibasic side chain at C11. These 2-substituted 5-methyl-indolo[2,3-b]quinoline derivatives were prepared by nucleophilic aromatic substitution $\left(\mathrm{S}_{\mathrm{N}} \mathrm{Ar}\right)$ of 11-chloroneocryptolepines with appropriate 1,2- and 1,3-diamines. Some of the 11-( $\omega$-aminoalkylamino) derivatives were further transformed into 11-ureido and thioureido analogues. Many of the prepared neocryptolepine derivatives showed submicromolar antiproliferative activity against the human leukemia MV4-11 cell line. Among them, 11-(3-amino-2-hydroxy)propylamino derivatives $\mathbf{2} \mathbf{h}$ and $\mathbf{2 k}$ were the most cytotoxic with a mean $\mathrm{IC}_{50}$ value of $0.042 \mu \mathrm{M}$ and $0.057 \mu \mathrm{M}$ against the MV4-11 cell line, $0.197 \mu \mathrm{M}$ and $0.1988 \mu \mathrm{M}$ against the A549 cell line, and $0.138 \mu \mathrm{M}$ and $0.117 \mu \mathrm{M}$ against the BALB/3T3 cell line, respectively.
\end{abstract}

Keywords: indolo[2,3-b]quinolone; neocryptolepine; antiproliferative activity; SAR study; aminoalkylamino-substituted

\section{Introduction}

The tetracyclic indoloquinoline ring systems constitute important structural motifs in natural products exhibiting numerous biological activities [1-3]. For example, cryptolepine (I, indolo[3,2-b]quinoline) and neocryptolepine (II, indolo[2,3-b]quinoline) are representative alkaloids 
isolated from the roots of the African plant Cryptolepis sanguinolenta [4-6] (Figure 1). Notably, an aqueous macerate or decoction of this plant is used in traditional medicine against malaria [7]. These two alkaloids, which only differ in the respective orientation of their indole and quinoline components, display potent antiparasitic properties [5,8-10]. Due to the linearly arranged planar tetracyclic structure, cryptolepine (I) and neocryptolepine (II) are DNA-intercalating agents and inhibit topoisomerase II, showing a high level of cytotoxicity [11-14].<smiles>Cn1c2c3ccccc3nc-2cc2ccccc21</smiles>

Cryptolepine (I)

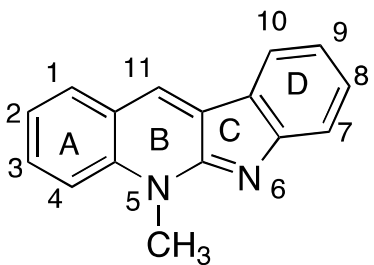

Neocryptolepine (II)

Figure 1. Chemical structures of indoloquinolines from Cryptolepis sanguinolenta.

Our previous structure activity relationship (SAR) study about the antiproliferative activity of the 5-methyl-indolo[2,3-b]quinoline derivatives revealed that the $\omega$-aminoalkylamino substituent at $\mathrm{C} 11$ is an important element for their bioactivity. For example, the 3-aminopropylamino group on 2 $\left(\mathrm{R}^{1}=\mathrm{H}\right)$ could increase the antiproliferative activity against the human leukemia cell line MV4-11 by about 20 times compared to that the of 11-chloro precursor $\mathbf{1}\left(\mathrm{R}^{1}=\mathrm{H}\right)[15,16]$.

Based on these findings, in this work, we further discuss the effect of the $\omega$-aminoalkylamino substituent at $\mathrm{C} 11$ in the 5-methyl-indolo[2,3-b]quinolone core by diversifying the side-chain structure, i.e., changing the length and branching of the linker between the two nitrogen atoms, etc. We also examine whether a hydroxy group residing on the spacer exerts influence on the antiproliferative activity.

\section{Results and Discussion}

\subsection{Synthesis}

The synthetic strategy for the 5-methyl-indolo[2,3-b]quinoline derivatives $\mathbf{2}$ is based on the nucleophilic aromatic substitution $\left(\mathrm{S}_{\mathrm{N}} \mathrm{Ar}\right)$ reaction of 11-chloroneocryptolepines $\mathbf{1}$ with an appropriate amine, as shown in Figure 2 [17]. The 11-chloroneocryptolepines 1, the key intermediates for the diversification, were derived starting with various substituted $\mathrm{N}$-methylanilines and methyl- $1 H$-indole-3-carboxylates in three steps in good yields [18]. The amination of 1 with various 1,2-diaminoethanes and 1,3-diaminopropanes using an excess amount of dimethyl formamide (DMF), under heating, smoothly yielded the corresponding 11 -aminated compounds $\mathbf{2}$. Subsequently, a series of ureido derivatives 3 and thioureido derivatives 4 were prepared in high yields by modification of the free terminal amine group of $\mathbf{2}$ with phenylisocyanate and isothiocyanate in dry $\mathrm{CH}_{2} \mathrm{Cl}_{2}$ at room temperature, respectively [19]. 


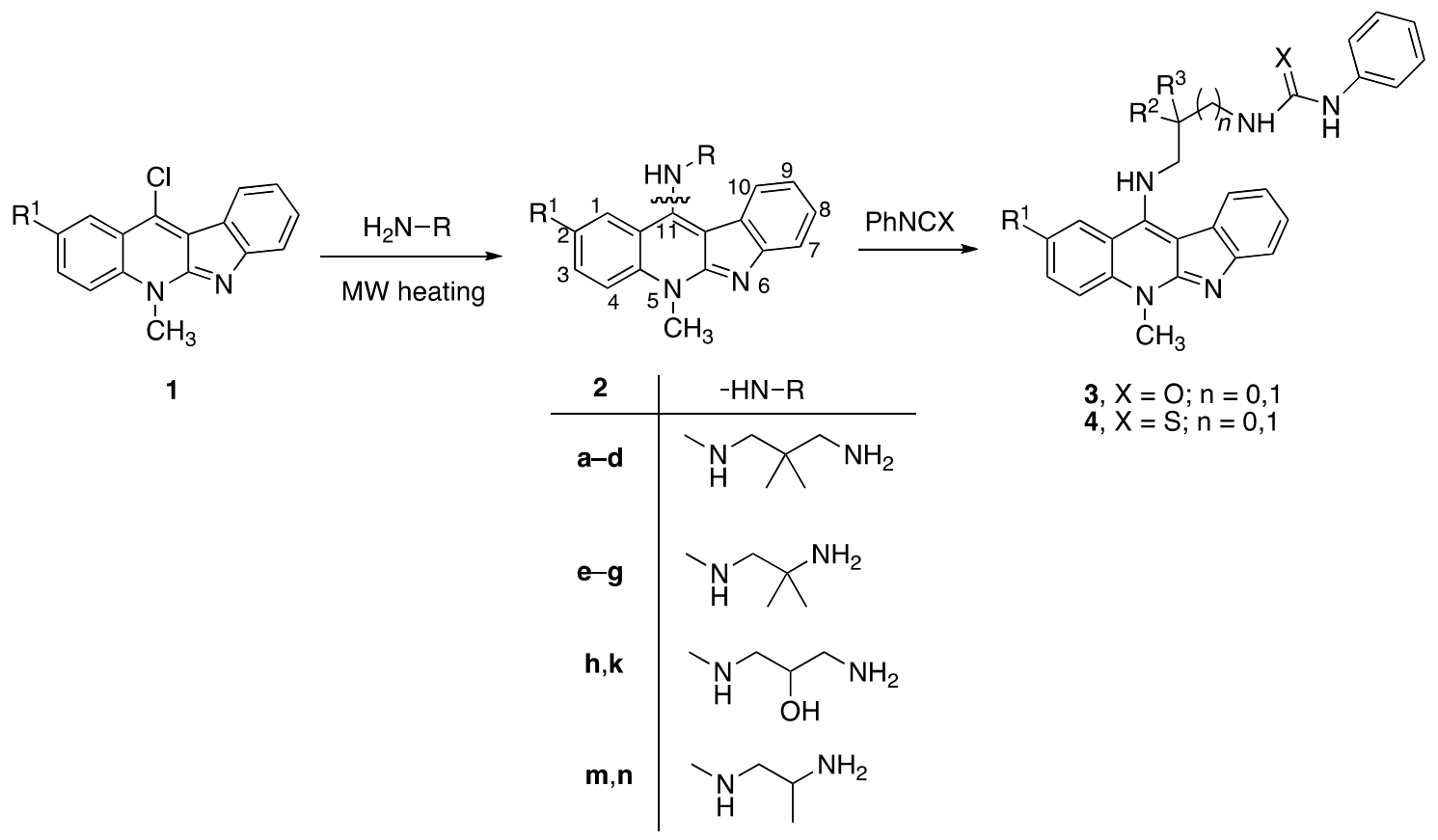

Figure 2. Synthesis of 11-aminoalkylamino-5-methyl-indolo[2,3-b]quinolines 2 and their ureido and thioureido derivatives 3 and 4 .

\subsection{Biological Evaluation}

The natural product neocryptolepine (II) is known to exhibit prominent antiproliferative activities, for example, $\mathrm{IC}_{50} 12.7 \pm 1.3 \mu \mathrm{M}$ against the human (HL-60) leukaemia cell line [14] and $\mathrm{IC}_{50}$ $7.48 \pm 4.42 \mu \mathrm{M}$ against the human breast cancer MDA-MB-453 cell line [20], which are comparable with the reference anticancer drug cisplatin $\left(\mathrm{IC}_{50} 7.6 \pm 0.7 \mu \mathrm{M}\right)$. This antiproliferative activity is improved slightly by introduction of the $\mathrm{Cl}$ atom at the $\mathrm{C} 11$ of neocryptolepine core to $\mathrm{IC}_{50} 5.16 \mu \mathrm{M}$. Introduction of an amino group is expected to increase the cytotoxic activity compared to the non-substituted neocryptolepine. Accordingly, various kinds of amino groups were then evaluated to assess their antiproliferative activity.

The synthesized compounds $\mathbf{2 a}-\mathbf{2 n}$ bearing various $\boldsymbol{\omega}$-aminoalkylamino groups at C11 were tested against the human leukemia MV4-11 cell line while varying substituents $\mathrm{R}^{1}$ at $\mathrm{C} 2$. As shown in Table 1, all assayed compounds were cytotoxic against MV4-11 leukemia cells ( $\mathrm{IC}_{50}$ values below $0.9 \mu \mathrm{M})$, and exhibited higher antiproliferative activities than the reference anticancer drug cisplatin ( $\left.\mathrm{IC}_{50} 2.82 \mu \mathrm{M}\right)$, but lower activities than doxorubicin $\mathrm{HCl}\left(\mathrm{IC}_{50} 0.006 \mu \mathrm{M}\right)$.

Amongst compounds $\mathbf{2 a - 2} \mathbf{d}$ bearing a geminal dimethyl group in the pendant on the amino side chain of the three-carbon-atoms spacer, the $\mathrm{Cl}$ atom at $\mathrm{C} 2$ was favored over the methyl group (i.e., $2 \mathrm{c}$ vs. $\mathbf{2 b}$ ) and the $\mathrm{Br}$ atom (i.e., $\mathbf{2 c}$ vs. $\mathbf{2 d}$ ). However, amongst compounds $\mathbf{2 e - 2} \mathbf{g}$ with a two-carbon-atoms spacer, compound $\mathbf{2} \mathbf{f}$ bearing a methyl group at $\mathrm{C} 2$ was more effective than $\mathbf{2 g}$ with a $\mathrm{Cl}$ atom at $\mathrm{C} 2$. Compound $\mathbf{2 j}$, bearing only one methyl group in the side chain on the two-carbon-atoms spacer, was more active than $\mathbf{2 g}$ with a geminal methyl group in the side chain. Compounds $\mathbf{2 m}$ and $\mathbf{2 n}$ bearing a $\mathrm{Cl}$ or a $\mathrm{Br}$ atom at $\mathrm{C} 2$, respectively, showed almost the same activity. Amongst the tested compounds, 2h, modified with a 3-amino-2-hydroxypropylamino group at $\mathrm{C} 11$ and a $\mathrm{Cl}$ at $\mathrm{C} 2$, showed the highest antiproliferative activity $\left(\mathrm{IC}_{50} 0.042 \mu \mathrm{M}\right)$. 
Table 1. Antiproliferative activity of 11-aminoalkylamino-5-methyl-indolo[2,3- $b$ ]quinolines against the human leukemia MV4-11 cell line.

\begin{tabular}{|c|c|c|c|c|}
\hline Compound & $\mathbf{R}^{1}$ & C11-Substituent & Yield, $\%$ of Amination & $\mathrm{IC}_{50}(\mu \mathrm{M})$ \\
\hline Cisplatin & & - & & $2.820 \pm 0.450$ \\
\hline Doxorubicin $\mathrm{HCl}$ & & . & - & $0.006 \pm 0.002$ \\
\hline $1 \mathrm{a}$ & $\mathrm{H}$ & $\mathrm{Cl}$ & - & $1.312 \pm 0.262$ \\
\hline $1 b$ & $\mathrm{Br}$ & $\mathrm{Cl}$ & - & $0.810 \pm 0.145$ \\
\hline $2 a$ & $\mathrm{H}$ & & 84 & $0.150 \pm 0.060$ \\
\hline $2 b$ & $\mathrm{Me}$ & & 86 & $0.288 \pm 0.075$ \\
\hline $2 c$ & $\mathrm{Cl}$ & $\mathrm{NH}$ & 95 & $0.119 \pm 0.043$ \\
\hline $2 d$ & $\mathrm{Br}$ & & 86 & $0.308 \pm 0.102$ \\
\hline $2 e$ & $\mathrm{H}$ & & 92 & $0.392 \pm 0.188$ \\
\hline $2 f$ & $\mathrm{Me}$ & & 88 & $0.105 \pm 0.027$ \\
\hline $2 \mathrm{~g}$ & $\mathrm{Cl}$ & & 90 & $0.453 \pm 0.209$ \\
\hline $2 \mathrm{~h}$ & $\mathrm{Cl}$ & $\mathrm{H}$ & 82 & $0.042 \pm 0.014$ \\
\hline $2 k$ & $\mathrm{Br}$ & & 76 & $0.057 \pm 0.015$ \\
\hline $2 \mathrm{~m}$ & $\mathrm{Cl}$ & & 87 & $0.103 \pm 0.014$ \\
\hline $2 n$ & $\mathrm{Br}$ & & 90 & $0.078 \pm 0.020$ \\
\hline
\end{tabular}

The $\mathrm{IC}_{50}$ value is defined as the concentration of a compound that corresponds to a $50 \%$ growth inhibition. Data are expressed as the mean $\pm \mathrm{SD}$.

Subsequently, the ureido and thioureido derivatives 3 and 4 were tested for their antiproliferative activity against the human leukemia MV4-11 cell line. Unfortunately, compounds 3 and 4 showed slightly less pronounced antiproliferative activities, compared with compound 2 , bearing a free terminal amino group (Table 2).

Table 2. Antiproliferative activity of the neocryptolepine derivatives $\mathbf{3 a}-\mathbf{3 d} \mathbf{d}, \mathbf{4 a}$, and $\mathbf{4} \mathbf{b}$ against the human leukemia MV4-11 cell line.

\begin{tabular}{ccccccc}
\hline Compound & $\mathbf{n}$ & $\mathbf{R}^{\mathbf{1}}$ & $\mathbf{R}^{\mathbf{2}}$ & $\mathbf{R}^{\mathbf{3}}$ & Yield, $\%$ & IC $_{\mathbf{5 0}}(\boldsymbol{\mu} \mathbf{M})$ \\
\hline 3a & 1 & $\mathrm{H}$ & $\mathrm{Me}$ & $\mathrm{Me}$ & $91^{\mathrm{a}}$ & $0.549 \pm 0.108$ \\
3b & 1 & $\mathrm{Me}$ & $\mathrm{Me}$ & $\mathrm{Me}$ & $83^{\mathrm{a}}$ & $0.427 \pm 0.092$ \\
3c & 1 & $\mathrm{Cl}$ & $\mathrm{Me}$ & $\mathrm{Me}$ & $84^{\mathrm{a}}$ & $0.790 \pm 0.302$ \\
3d & 0 & $\mathrm{H}$ & $\mathrm{Me}$ & $\mathrm{Me}$ & $89^{\mathrm{a}}$ & $0.464 \pm 0.141$ \\
$\mathbf{4 a}$ & 1 & $\mathrm{H}$ & $\mathrm{Me}$ & $\mathrm{Me}$ & $90^{\mathrm{b}}$ & $0.680 \pm 0.215$ \\
$\mathbf{4 b}$ & 0 & $\mathrm{Cl}$ & $\mathrm{Me}$ & $\mathrm{Me}$ & $84^{\mathrm{b}}$ & $2.330 \pm 1.015$ \\
\hline
\end{tabular}

a Yield of ureidation of the precursor amine 2 ; $^{\mathrm{b}}$ Yield of thioureidation of the precursor amine 2 . The $\mathrm{IC}_{50}$ value is defined as the concentration of a compound that corresponds to a 50\% growth inhibition. Data are expressed as the mean \pm SD.

Compounds $\mathbf{2 a}, \mathbf{2} \mathbf{c}, \mathbf{2 f}, \mathbf{2 h}, \mathbf{2 k}, \mathbf{2 m}$, and $\mathbf{2 n}$ being of high antiproliferative activity against MV4-11 cells were chosen as candidates for further studies on their antiproliferative activity against 
non-small cell lung cancer (A549) and colon cancer (HCT116) cell lines, along with normal murine fibroblasts (BALB/3T3). The test results are listed in Table 3. All tested compounds showed higher antiproliferative activities against the cancer cells than the cisplatin used as a control agent. Compounds 2k and $\mathbf{2 h}$ showed higher activity against the A549 and HCT116 cell line than other compounds, and they showed selective antiproliferative activity against the MV4-11 cell line. The 2-bromo-derivative 2n and the 2-chloro-derivative $\mathbf{2 m}$ showed almost the same cytotoxicity against A549 and HCT116 cell lines compared to the normal cell line BALB/3T3, but 2 n showed potent activity against the MV4-11 cell line with an $\mathrm{IC}_{50}$ value of $0.078 \mu \mathrm{M}$. Compounds $\mathbf{2 c}$ and $\mathbf{2 f}$ showed lower cytotoxicity against the normal cell line, and moderate activity against the A549 and HCT116 cell lines. It is quite obvious that the introduction of proper alkylamino substituents into biologically active derivatives can favourably influence their activities and selectivities in DNA binding.

Table 3. Antiproliferative activity of 11-alkylaminated 5-methyl-indolo[2,3-b]quinolines against normal mice fibroblasts BALB/3T3 and against the cancer cell lines A549 and HCT 116.

\begin{tabular}{cccc}
\hline Compound & ${\text { BALB/3T3 } \mathbf{I C}_{\mathbf{5 0}}(\boldsymbol{\mu M})}$ & ${\text { A 549 } \text { IC }_{\mathbf{5 0}}(\boldsymbol{\mu M})}$ & HCT 116 IC $_{\mathbf{5 0}}(\boldsymbol{\mu M})$ \\
\hline Cisplatin & $8.700 \pm 0.097$ & $9.870 \pm 2.400$ & $8.500 \pm 0.540$ \\
Doxorubicin HCl & $1.078 \pm 0.033$ & $0.329 \pm 0.097$ & $0.390 \pm 0.098$ \\
$\mathbf{2 a}$ & $4.789 \pm 2.018$ & $1.512 \pm 0.198$ & $1.262 \pm 0.361$ \\
$\mathbf{2 c}$ & $9.131 \pm 0.844$ & $1.455 \pm 0.168$ & $1.373 \pm 0.351$ \\
$\mathbf{2 f}$ & $10.558 \pm 0.330$ & $1.795 \pm 0.270$ & $2.370 \pm 0.481$ \\
$\mathbf{2 h}$ & $0.896 \pm 0.042$ & $0.197 \pm 0.028$ & $0.138 \pm 0.050$ \\
$\mathbf{2 k}$ & $0.864 \pm 0.015$ & $0.190 \pm 0.027$ & $0.117 \pm 0.055$ \\
$\mathbf{2 m}$ & $1.018 \pm 0.017$ & $1.269 \pm 0.118$ & $1.204 \pm 0.283$ \\
$\mathbf{2 n}$ & $0.939 \pm 0.018$ & $0.988 \pm 0.164$ & $0.842 \pm 0.367$ \\
\hline
\end{tabular}

The $\mathrm{IC}_{50}$ value is defined as the concentration of a compound that corresponds to a $50 \%$ growth inhibition. Human lung cancer cell line (A-549); human colon adenocarcinoma cell line (HCT 116); normal murine embryonic fibroblast cell line (BALB/3T3). Data are expressed as the mean \pm SD.

\subsection{Spectroscopic Characterization of Neocryptolepine Derivative 2d Interacting with Salmon Sperm DNA}

The DNA binding studies of compound $\mathbf{2 d}$ were performed using UV-Vis absorption spectroscopy with salmon sperm DNA in a phosphate buffer of pH 7.0 at $20{ }^{\circ} \mathrm{C}$. A red shift and a hypochromic effect were observed in the absorption spectra while the DNA solution was gradually added to the solution of compound $\mathbf{2 d}$. The results depleted in Figure 3a showed that the minimum of the absorption band at $256 \mathrm{~nm}$ for $\mathbf{2 d}$ decreased while increasing the DNA concentration. The maximum absorption shifted from $256 \mathrm{~nm}$ to $289 \mathrm{~nm}$. This illustrated that the mode of $\mathbf{2 d}$ binding to DNA was intercalation. The binding constant of $2 \mathrm{~d}$ to DNA was calculated as $4.12 \times 10^{5} \mathrm{~L} \cdot \mathrm{mol}^{-1}$, according to a double-reciprocal equation.

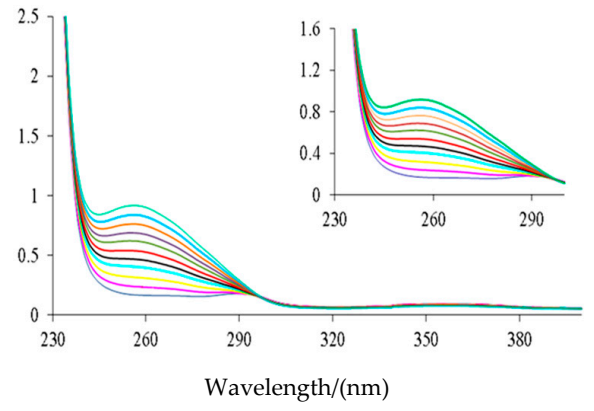

(a)

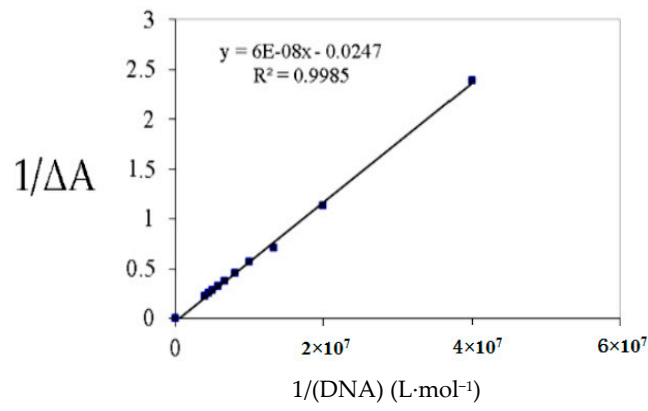

(b)

Figure 3. (a) UV-Vis absorption spectra of compound $\mathbf{2 d}$ at $20^{\circ} \mathrm{C} . \mathrm{C}(\mathbf{2 d})=50 \mu \mathrm{mol} / \mathrm{L}, \mathrm{C}(\mathrm{DNA})=0.0$, $0.025,0.05,0.075,0.1,0.125,0.15,0.175,0.2,0.225,0.25 \mu \mathrm{mol} / \mathrm{L}$ for curves $1-11$ in phosphate buffer solution (pH 7.0) (b) Plot of $1 / \Delta$ A vs. 1/[DNA] for 2d-DNA. 


\section{Experimental}

\subsection{Chemistry}

The commercially obtained reagents (TCI, Tokyo, Japan) were used directly without further purification. The ${ }^{1} \mathrm{H}-\mathrm{NMR}$ and ${ }^{13} \mathrm{C}-\mathrm{NMR}$ spectra were taken on Varian INOVA-600, Varian INOVA-400, Varian INOVA-300 (Palo Alto, CA, USA), or Bruker 400 spectrometers (Billerica, MA, USA), using $\mathrm{CDCl}_{3}$ or DMSO- $d_{6}$ as a solvent and tetramethylsilane (TMS) as the internal standard. The NMR spectra of the compounds are in Supplementary Materials. Mass spectra were obtained on a Bruker microTOF II-SKA spectrometer. The melting points were determined on a RFS-10 hot stage microscope (J-Science, Kyoto, Japan). The UV spectra were recorded with a U-2910 spectrophotometer (Hitachi, Tokyo, Japan) in a $1 \mathrm{~cm}$ path length quartz cuvette at an indicated wavelength, and the sample was dissolved in phosphate buffer with $2.5 \%$ DMSO.

\subsubsection{General Procedure for the Synthesis of 11-Aminoneocryptolepines $\mathbf{2 a}-\mathbf{2 n}$}

11-Chloroindoloquinolines $\mathbf{1}(0.3 \mathrm{mmol})$ and an excess of the appropriate $\omega$-aminoalkylamine $(3.0 \mathrm{mmol})$ were heated together at $120^{\circ} \mathrm{C}$ for $4 \mathrm{~h}$. Thin Layer Chromatography (TLC) monitoring was used to ensure the completion of the reaction. The resulting brown crude oil was purified by flash chromatography using AcOEt/2 $\mathrm{M}$ ammonia in $\mathrm{MeOH}(9: 1, v / v)$ as an eluent to yield pure yellowish solid products.

N-(3-Amino-2,2-dimethylpropyl)-5-methyl-5H-indolo[2,3-b]quinolin-11-amine 2a. Yellowish solid. Yield: $84 \%$. M.p. $112-114{ }^{\circ} \mathrm{C} .{ }^{1} \mathrm{H}-\mathrm{NMR}\left(400 \mathrm{MHz}, \mathrm{CDCl}_{3}\right): \delta=8.18(\mathrm{dd}, J=8.0,4.0 \mathrm{~Hz}, 1 \mathrm{H}), 8.00(\mathrm{~d}, J=8.0 \mathrm{~Hz}$, $1 \mathrm{H}), 7.76(\mathrm{~d}, J=8.0 \mathrm{~Hz}, 1 \mathrm{H}), 7.69-7.61(\mathrm{~m}, 2 \mathrm{H}), 7.40(\mathrm{t}, J=8.0 \mathrm{~Hz}, 1 \mathrm{H}), 7.32-7.28(\mathrm{t}, J=8.0 \mathrm{~Hz}, 1 \mathrm{H})$, $7.16(\mathrm{t}, J=8.0 \mathrm{~Hz}, 1 \mathrm{H}), 4.23(\mathrm{~s}, 3 \mathrm{H}), 3.85(\mathrm{~s}, 2 \mathrm{H}), 2.86(\mathrm{~s}, 2 \mathrm{H}), 0.90(\mathrm{~s}, 6 \mathrm{H}) .{ }^{13} \mathrm{C}-\mathrm{NMR}\left(100 \mathrm{MHz}, \mathrm{CDCl}_{3}\right)$ : $\delta=157.1,152.2,149.2,138.1,130.0,125.0,124.4,121.7,120.3,118.5,117.1,116.1,114.6,105.0,61.2,53.0$, 36.0, 32.7, 23.6 (2C). HRMS (ESI): $m / z=331.4462[\mathrm{M}-\mathrm{H}]^{-}$, calcd. 332.4421.

N-(3-Amino-2,2-dimethylpropyl)-2,5-dimethyl-5H-indolo[2,3-b]quinolin-11-amine $\mathbf{2 b}$. Yellowish solid. Yield: $86 \%$ M.p. $173-175^{\circ} \mathrm{C} .{ }^{1} \mathrm{H}-\mathrm{NMR}\left(400 \mathrm{MHz}, \mathrm{CDCl}_{3}\right): \delta=8.01(\mathrm{br} \mathrm{s}, 1 \mathrm{H}), 7.98(\mathrm{~d}, J=8.0 \mathrm{~Hz}, 1 \mathrm{H}), 7.74(\mathrm{~d}$, $J=8.0 \mathrm{~Hz}, 1 \mathrm{H}), 7.57-7.50(\mathrm{~m}, 2 \mathrm{H}), 7.39(\mathrm{t}, J=8.0 \mathrm{~Hz}, 1 \mathrm{H}), 7.14(\mathrm{t}, J=8.0 \mathrm{~Hz}, 1 \mathrm{H}), 4.24(\mathrm{~s}, 3 \mathrm{H}), 3.84(\mathrm{~s}$, 2H), $2.87(\mathrm{~s}, 2 \mathrm{H}), 2.53(\mathrm{~s}, 3 \mathrm{H}) 0.95(\mathrm{~s}, 6 \mathrm{H}) .{ }^{13} \mathrm{C}-\mathrm{NMR}\left(100 \mathrm{MHz}, \mathrm{CDCl}_{3}\right): \delta=149.0,136.4,131.4,129.6$, 125.1, 124.4, 123.9, 121.5, 118.3, 117.2, 116.0, 114.5, 106.0, 60.6, 52.8, 36.1, 32.6, 23.6 (2C), 21.1. HRMS (ESI): $m / z=347.2204[\mathrm{M}+\mathrm{H}]^{-}$, calcd. 347.2236.

$\mathrm{N}$-(3-Amino-2,2-dimethylpropyl)-2-chloro-5-methyl-5H-indolo[2,3-b]quinolin-11-amine 2c. Yellowish solid. Yield: $95 \%$. M.p. $177-179{ }^{\circ} \mathrm{C} .{ }^{1} \mathrm{H}-\mathrm{NMR}\left(400 \mathrm{MHz}, \mathrm{CDCl}_{3}\right): \delta=8.20(\mathrm{~s}, 1 \mathrm{H}), 7.97(\mathrm{~d}, J=8 \mathrm{~Hz}, 1 \mathrm{H}), 7.76(\mathrm{~d}$, $J=8 \mathrm{~Hz}, 1 \mathrm{H}), 7.60-7.56(\mathrm{~m}, 2 \mathrm{H}), 7.41(\mathrm{~m}, 1 \mathrm{H}), 7.18(\mathrm{~m}, 1 \mathrm{H}), 4.22(\mathrm{~s}, 3 \mathrm{H}), 3.85(\mathrm{~s}, 2 \mathrm{H}), 2.92(\mathrm{~s}, 2 \mathrm{H}), 0.90(\mathrm{~s}$, 6H). ${ }^{13} \mathrm{C}-\mathrm{NMR}\left(100 \mathrm{MHz}, \mathrm{CDCl}_{3}\right): \delta=148.4,136.8,130.4(2 \mathrm{C}), 126.3,125.7(2 \mathrm{C}), 124.7,124.0(2 \mathrm{C}), 122.4$ (2C), 119.2, 117.5, 116.3, 62.0, 53.4, 36.0, 33.4, 24.0 (2C). HRMS (ESI): $m / z=365.1624[\mathrm{M}-\mathrm{H}]^{-}$, calcd. 366.1611.

N-(3-Amino-2,2-dimethylpropyl)-2-bromo-5-methyl-5H-indolo[2,3-b]quinolin-11-amine 2d. Yellowish solid. Yield: $86 \%$. M.p. $184-186{ }^{\circ} \mathrm{C} .{ }^{1} \mathrm{H}-\mathrm{NMR}\left(400 \mathrm{MHz}, \mathrm{CDCl}_{3}\right): \delta=7.97(\mathrm{q}, J=7.8 \mathrm{~Hz}, 2 \mathrm{H}), 7.76(\mathrm{~d}, J=7.8 \mathrm{~Hz}$, $1 \mathrm{H}), 7.55(\mathrm{q}, J=7.2 \mathrm{~Hz}, 1 \mathrm{H}), 7.40(\mathrm{t}, J=7.5 \mathrm{~Hz}, 1 \mathrm{H}), 7.26(\mathrm{t}, J=5.4 \mathrm{~Hz}, 1 \mathrm{H}), 7.19(\mathrm{t}, J=7.8 \mathrm{~Hz}, 1 \mathrm{H}), 4.06$ $(\mathrm{s}, 3 \mathrm{H}), 3.66(\mathrm{~s}, 2 \mathrm{H}), 2.68(\mathrm{~s}, 2 \mathrm{H}), 0.76(\mathrm{~s}, 6 \mathrm{H}) .{ }^{13} \mathrm{C}-\mathrm{NMR}\left(75 \mathrm{MHz}, \mathrm{CDCl}_{3}\right): \delta=157.1,152.4,148.0,136.8$, $132.7,126.7,125.4,124.8,122.5,118.9,117.7,117.4,116.3,113.3,104.6,62.0,53.2,35.7,33.1,23.9$ (2C).

$\mathrm{N}$-(2-Amino-2-methylpropyl)-5-methyl-5H-indolo[2,3-b]quinolin-11-amine 2e. Yellowish solid. Yield: 92\%. M.p. $130-132{ }^{\circ} \mathrm{C} .{ }^{1} \mathrm{H}-\mathrm{NMR}\left(400 \mathrm{MHz}, \mathrm{CDCl}_{3}\right): \delta=8.24(\mathrm{~d}, J=8.0 \mathrm{~Hz}, 1 \mathrm{H}), 8.14(\mathrm{~d}, J=4.0 \mathrm{~Hz}, 1 \mathrm{H})$, 7.80-7.71 (m, 3H), 7.43-7.41 (m, 1H), 7.33 (m, 1H), $7.01(\mathrm{~d}, J=8.0 \mathrm{~Hz}, 1 \mathrm{H}), 4.28(\mathrm{~s}, 3 \mathrm{H}), 3.68(\mathrm{~S}, 2 \mathrm{H})$, $1.13(\mathrm{~s}, 6 \mathrm{H}) .{ }^{13} \mathrm{C}-\mathrm{NMR}\left(100 \mathrm{MHz}, \mathrm{CDCl}_{3}\right): \delta=156.8,152.6,149.5,138.7,130.6,125.7,125.1,124.6,121.5$, 120.6, 119.2, 117.5, 115.8, 115.0, 106.9, 59.4, 51.6, 33.0, 29.4 (2C). HRMS (ESI): $m / z=319.1911\left[\mathrm{M}+\mathrm{H}^{-}{ }^{-}\right.$, calcd. 319.1923. 
N-(2-Amino-2-methylpropyl)-2,5-dimethyl-5H-indolo[2,3-b]quinolin-11-amine 2f. Yellowish solid. Yield: 88 \%. M.p. $132-134{ }^{\circ} \mathrm{C} .{ }^{1} \mathrm{H}-\mathrm{NMR}\left(400 \mathrm{MHz}, \mathrm{CDCl}_{3}\right): \delta=8.13(\mathrm{~d}, J=7.6 \mathrm{~Hz}, 1 \mathrm{H}), 7.95(\mathrm{~s}, 1 \mathrm{H}), 7.76(\mathrm{~d}$, $J=8.0 \mathrm{~Hz}, 1 \mathrm{H}), 7.53(\mathrm{q}, J=8.0 \mathrm{~Hz}, 2 \mathrm{H}), 7.42(\mathrm{t}, J=8.0 \mathrm{~Hz}, 1 \mathrm{H}), 7.19(\mathrm{t}, J=7.6 \mathrm{~Hz}, 1 \mathrm{H}), 6.71(\mathrm{~s}, 1 \mathrm{H}), 4.23$ $(\mathrm{s}, 3 \mathrm{H}), 3.64(\mathrm{~d}, J=8.0 \mathrm{~Hz}, 2 \mathrm{H}), 2.55(\mathrm{~s}, 3 \mathrm{H}), 1.10(\mathrm{~s}, 6 \mathrm{H}) .{ }^{13} \mathrm{C}-\mathrm{NMR}\left(101 \mathrm{MHz}, \mathrm{CDCl}_{3}\right): \delta=157.0,153.0$, 149.6, 137.0, 132.1, 130.2, 125.8, 124.7 (2C), 124.6, 121.6, 119.0, 117.5, 116.1, 115.1, 59.7, 51.9, $33.1,29.6$ (2C), 21.7. HRMS (ESI): $m / z=333.2054[\mathrm{M}+\mathrm{H}]^{-}$, calcd. 333.2079.

N-(2-Amino-2-methylpropyl)-2-chloro-5-methyl-5H-indolo[2,3-b]quinolin-11-amine 2g. Yellowish solid. Yield: 90\%. M.p. $108-110{ }^{\circ} \mathrm{C} .{ }^{1} \mathrm{H}-\mathrm{NMR}\left(400 \mathrm{MHz}, \mathrm{CDCl}_{3}\right): \delta=8.15(\mathrm{q}, J=7.6,2 \mathrm{H}), 7.76(\mathrm{~d}, J=8.0 \mathrm{~Hz}$, $1 \mathrm{H}), 7.62(\mathrm{~m}, 2 \mathrm{H}), 7.44(\mathrm{t}, J=7.6 \mathrm{~Hz}, 1 \mathrm{H}), 7.21(\mathrm{t}, J=7.6 \mathrm{~Hz}, 1 \mathrm{H}), 6.73(\mathrm{br}, 1 \mathrm{H}), 4.22(\mathrm{~s}, 3 \mathrm{H}), 3.58$ $(\mathrm{d}, J=4.5 \mathrm{~Hz}, 2 \mathrm{H}), 1.11(\mathrm{~s}, 6 \mathrm{H}) .{ }^{13} \mathrm{C}-\mathrm{NMR}\left(101 \mathrm{MHz}, \mathrm{CDCl}_{3}\right): \delta=156.4,152.9,148.2,137.1,130.4$, $126.1,125.9,124.5,124.4,121.6,119.4,117.6,116.8,116.4,107.6,59.1,51.5,33.1,29.5$ (2C). HRMS (ESI): $m / z=351.1422[\mathrm{M}-\mathrm{H}]^{-}$, calcd. 352.1455.

1-Amino-3-(2-chloro-5-methyl-5H-indolo[2,3-b]quinolin-11-ylamino)propan-2-ol 2h. Yellowish solid. Yield: 82\%. M.p. $150-152{ }^{\circ} \mathrm{C} .{ }^{1} \mathrm{H}-\mathrm{NMR}\left(400 \mathrm{MHz}, \mathrm{DMSO}-\mathrm{d}_{6}\right): \delta=8.56(\mathrm{~s}, 1 \mathrm{H}), 7.95(\mathrm{~d}, J=7.6 \mathrm{~Hz}, 1 \mathrm{H}), 7.85(\mathrm{~d}$, $J=9.2 \mathrm{~Hz}, 1 \mathrm{H}), 7.78(\mathrm{q}, J=4 \mathrm{~Hz}, 1 \mathrm{H}), 7.50(\mathrm{~d}, J=8 \mathrm{~Hz}, 1 \mathrm{H}), 7.28(\mathrm{t}, J=8 \mathrm{~Hz}, 1 \mathrm{H}), 7.07(\mathrm{q}, J=8 \mathrm{~Hz}, 1 \mathrm{H})$, $4.13(\mathrm{~s}, 3 \mathrm{H}), 3.91-3.87(\mathrm{~m}, 1 \mathrm{H}), 3.79(\mathrm{dd}, J=12.8,6.5 \mathrm{~Hz}, 2 \mathrm{H}), 3.69-3.66(\mathrm{~m}, 2 \mathrm{H}) .{ }^{13} \mathrm{C}-\mathrm{NMR}(101 \mathrm{MHz}$, DMSO- $\left.d_{6}\right): \delta=156.1,152.4,147.5,136.1,130.1,124.9(2 \mathrm{C}), 123.9,123.4,122.2,118.1,117.0,116.7,116.6$, 105.4, 70.9, 52.3, 45.4, 32.3. HRMS (ESI): $m / z=354.1247[\mathrm{M}-\mathrm{H}]^{-}$, calcd. 353.11275.

1-Amino-3-(2-bromo-5-methyl-5H-indolo[2,3-b]quinolin-11-ylamino)propan-2-ol 2k. Yellowish solid. Yield: 76\%. M.p. $162-164{ }^{\circ} \mathrm{C} .{ }^{1} \mathrm{H}-\mathrm{NMR}\left(400 \mathrm{MHz}, \mathrm{DMSO}-d_{6}\right): \delta=8.67(\mathrm{~s}, 1 \mathrm{H}), 7.94(\mathrm{t}, J=8.0 \mathrm{~Hz}, 2 \mathrm{H}), 7.81$ $(\mathrm{d}, J=8.0 \mathrm{~Hz}, 1 \mathrm{H}), 7.52(\mathrm{~d}, J=8.0,1 \mathrm{H}), 7.30(\mathrm{t}, J=7.8 \mathrm{~Hz}, 1 \mathrm{H}), 7.11(\mathrm{t}, J=8.0 \mathrm{~Hz}, 1 \mathrm{H}), 4.13(\mathrm{~s}, 3 \mathrm{H})$, 3.87-3.82 (m, 1H), $3.81(\mathrm{dd}, J=12.0,6.5 \mathrm{~Hz}, 2 \mathrm{H}), 3.68-3.65(\mathrm{~m}, 2 \mathrm{H}) .{ }^{13} \mathrm{C}-\mathrm{NMR}\left(101 \mathrm{MHz}, \mathrm{DMSO}-d_{6}\right)$ : $\delta=156.1,152.4,147.5,136.4,132.8,126.3$ (2C), 124.9, 123.9, 122.2, 118.2, 117.3, 116.7, 112.7, 105.2, 70.9, $52.3,45.4,32.3$.

N-(2-Aminopropyl)-2-chloro-5-methyl-5H-indolo[2,3-b]quinolin-11-amine 2m. Yellowish solid. Yield: 87\%. M.p. $95-79{ }^{\circ} \mathrm{C} .{ }^{1} \mathrm{H}-\mathrm{NMR}\left(600 \mathrm{MHz}, \mathrm{CDCl}_{3}\right): \delta=8.10(\mathrm{~d}, J=6 \mathrm{~Hz}, 2 \mathrm{H}), 7.76(\mathrm{~d}, J=7.8 \mathrm{~Hz}, 1 \mathrm{H}), 7.59$ (t, $J=6.6 \mathrm{~Hz}, 1 \mathrm{H}), 7.54(\mathrm{~d}, J=9.1 \mathrm{~Hz}, 1 \mathrm{H}), 7.44(\mathrm{t}, J=7.6 \mathrm{~Hz}, 1 \mathrm{H}), 7.22(\mathrm{t}, J=7.2 \mathrm{~Hz}, 1 \mathrm{H}), 6.48(\mathrm{br}, 1 \mathrm{H}), 4.20$ $(\mathrm{s}, 3 \mathrm{H}), 3.79(\mathrm{~d}, J=11.4 \mathrm{~Hz}, 1 \mathrm{H}), 3.40(\mathrm{t}, J=10.8 \mathrm{~Hz}, 1 \mathrm{H}), 3.12(\mathrm{q}, J=6 \mathrm{~Hz}, 1 \mathrm{H}), 1.10(\mathrm{~d}, J=6.2 \mathrm{~Hz}, 3 \mathrm{H})$. ${ }^{13} \mathrm{C}-\mathrm{NMR}\left(151 \mathrm{MHz} \mathrm{CDCl}_{3}\right): \delta=156.3,152.8,147.9,137.0,130.4,126.2,125.9,124.5,124.3,121.5,119.4$, 117.6, 116.9, 116.3, 108.1, 55.1, 47.9, 33.1, 22.9. HRMS (ESI): $m / z=337.1232[\mathrm{M}-\mathrm{H}]^{-}$, calcd. 338.1298.

N-(2-Amino-2-methylpropyl)-2-bromo-5-methyl-5H-indolo[2,3-b]quinolin-11-amine 2n. Yellowish solid. Yield: 90\%. M.p. $101-103{ }^{\circ} \mathrm{C} .{ }^{1} \mathrm{H}-\mathrm{NMR}\left(600 \mathrm{MHz}, \mathrm{CDCl}_{3}\right): \delta=8.21(\mathrm{~s}, 1 \mathrm{H}), 8.08(\mathrm{~d}, J=7.8 \mathrm{~Hz}, 1 \mathrm{H}), 7.74$ $(\mathrm{d}, J=7.8 \mathrm{~Hz}, 1 \mathrm{H}), 7.71(\mathrm{q}, J=6 \mathrm{~Hz}, 1 \mathrm{H}), 7.48-7.43(\mathrm{~m}, 2 \mathrm{H}), 7.20(\mathrm{t}, J=7.8 \mathrm{~Hz}, 1 \mathrm{H}), 6.46(\mathrm{~s}, 1 \mathrm{H}), 4.15(\mathrm{~s}$, $3 \mathrm{H}), 3.74(\mathrm{~d}, J=11.4 \mathrm{~Hz}, 1 \mathrm{H}), 3.37(\mathrm{dd}, J=7.8,7.8 \mathrm{~Hz}, 1 \mathrm{H}), 3.11-3.08(\mathrm{~m}, 1 \mathrm{H}), 1.09(\mathrm{~d}, J=6.0 \mathrm{~Hz}, 3 \mathrm{H})$. ${ }^{13} \mathrm{C}-\mathrm{NMR}\left(75 \mathrm{MHz} \mathrm{CDCl}_{3}\right): \delta=156.1,152.6,147.8,137.3,133.1,127.5,127.2,126.2,124.2,121.5,119.4$, $117.6,116.6,113.3,108.0,55.1,47.9,33.2,22.9$.

\subsubsection{General Procedure for the Synthesis of $\mathbf{3 a}-\mathbf{3 d}$ and $\mathbf{4 a}, \mathbf{b}$}

2-Substituted 5-methyl-5H-indolo]2,3-b]quinolone-11-amine (2) (50 mg) was dissolved in dry $\mathrm{CH}_{2} \mathrm{Cl}_{2}(1 \mathrm{~mL})$, and a solution of phenylisocyanate or phenylisothiocyanate (1.1 equiv.) in $\operatorname{dry} \mathrm{CH}_{2} \mathrm{Cl}_{2}$ $(1 \mathrm{~mL})$ was added drop by drop under stirring at room temperature for $2-4 \mathrm{~h}$. TLC monitoring was used to ensure the completion of the reaction. The reaction mixture was evaporated to dryness under reduced pressure. The crude product was purified by flash chromatography using AcOEt/2M ammonia in $\mathrm{MeOH}(9: 1, v / v)$ as an eluent to yield pure products as yellowish solids.

1-(2,2-Dimethyl-3-(5-methyl-5H-indolo[2,3-b]quinolin-11-ylamino)propyl)-3-phenylurea 3a. Yellowish solid. Yield: 91\%. M.p. $154-156{ }^{\circ} \mathrm{C} .{ }^{1} \mathrm{H}-\mathrm{NMR}\left(600 \mathrm{MHz}, \mathrm{CDCl}_{3}\right): \delta=9.25(\mathrm{~s}, 1 \mathrm{H}), 8.42(\mathrm{~d}, J=6 \mathrm{~Hz}, 1 \mathrm{H})$, 7.74-7.70 (m, 2H), $7.64(\mathrm{~d}, J=6 \mathrm{~Hz}, 1 \mathrm{H}), 7.46(\mathrm{~s}, 2 \mathrm{H}), 7.40-7.35(\mathrm{~m}, 2 \mathrm{H}), 7.24-7.22(\mathrm{~m}, 2 \mathrm{H}), 7.14(\mathrm{~s}, 1 \mathrm{H})$, 
$6.97(\mathrm{~s}, 1 \mathrm{H}), 6.77(\mathrm{~s}, 1 \mathrm{H}), 6.64(\mathrm{~s}, 1 \mathrm{H}), 4.03(\mathrm{~s}, 3 \mathrm{H}), 3.60(\mathrm{~s}, 2 \mathrm{H}), 3.25(\mathrm{~s}, 2 \mathrm{H}), 2.29(\mathrm{br}, 1 \mathrm{H}), 0.63(\mathrm{~s}, 6 \mathrm{H})$. ${ }^{13} \mathrm{C}-\mathrm{NMR}\left(151 \mathrm{MHz}, \mathrm{CDCl}_{3}\right): \delta=158.0,157.1,151.5,149.6,140.0,137.9,130.9,129.5(2 \mathrm{C}), 126.0,124.4$, 124.2, 123.1, 122.6, 121.9, 120.0 (2C), 119.7, 116.9, 116.5, 115.0, 106.8, 55.7, 48.0, 38.6, 33.3, 24.0 (2C). HRMS (ESI): $m / z=450.2364[\mathrm{M}-\mathrm{H}]^{-}$, calcd. 451.2372 .

1-(3-(2,5-Dimethyl-5H-indolo[2,3-b]quinolin-11-ylamino)-2,2-dimethylpropyl)-3-phenylurea $\mathbf{3 b}$. Yellowish solid. Yield: $83 \%$. M.p. $228-230{ }^{\circ} \mathrm{C} .{ }^{1} \mathrm{H}-\mathrm{NMR}\left(300 \mathrm{MHz}, \mathrm{CDCl}_{3}\right): \delta=9.18(\mathrm{~s}, 1 \mathrm{H}), 8.12(\mathrm{~s}, 1 \mathrm{H}), 7.67$ $(\mathrm{d}, J=7.8 \mathrm{~Hz}, 1 \mathrm{H}), 7.57(\mathrm{~d}, J=7.8 \mathrm{~Hz}, 1 \mathrm{H}), 7.45(\mathrm{~d}, J=9 \mathrm{~Hz}, 3 \mathrm{H}), 7.34(\mathrm{q}, J=6 \mathrm{~Hz}, 2 \mathrm{H}), 7.23(\mathrm{~d}$, $J=9 \mathrm{~Hz}, 2 \mathrm{H}), 7.08(\mathrm{t}, J=9 \mathrm{~Hz}, 1 \mathrm{H}), 6.98(\mathrm{t}, J=6 \mathrm{~Hz}, 1 \mathrm{H}), 6.49(\mathrm{~s}, 1 \mathrm{H}), 6.10(\mathrm{~s}, 1 \mathrm{H}), 3.97(\mathrm{~s}, 3 \mathrm{H}), 3.52(\mathrm{~d}$, $J=6.6 \mathrm{~Hz}, 2 \mathrm{H}), 3.35(\mathrm{~d}, J=6.2 \mathrm{~Hz}, 2 \mathrm{H}), 2.58(\mathrm{~s}, 3 \mathrm{H}), 0.77(\mathrm{~s}, 6 \mathrm{H}) .{ }^{13} \mathrm{C}-\mathrm{NMR}\left(101 \mathrm{MHz}, \mathrm{CDCl}_{3}\right): \delta=157.9$, 156.4, 150.7, 149.7, 140.3, 136.0, 132.5, 131.6, 129.4 (2C), 125.9, 124.2 (2C), 124.0, 122.8, 122.6, 119.6 (2C), 116.8, 116.2, 114.9, 106.5, 55.7, 48.0, 38.6, 33.3, 24.1(2C), 21.7. HRMS (ESI): $m / z=466.4585\left[\mathrm{M}+\mathrm{H}^{-}\right.$, calcd. 466.2607 .

1-(3-(2-Chloro-5-methyl-5H-indolo[2,3-b]quinolin-11-ylamino)-2,2-dimethylpropyl)-3-phenylurea 3c. Yellowish solid. Yield: $84 \%$. M.p. $232-234{ }^{\circ} \mathrm{C} .{ }^{1} \mathrm{H}-\mathrm{NMR}\left(400 \mathrm{MHz}, \mathrm{CDCl}_{3}\right): \delta=8.36(\mathrm{~s}, 1 \mathrm{H}), 8.13(\mathrm{br}$, $1 \mathrm{H}), 7.73-7.70(\mathrm{~m}, 2 \mathrm{H}), 7.60-7.57(\mathrm{~m}, 1 \mathrm{H}), 7.45-7.39(\mathrm{~m}, 3 \mathrm{H}), 7.39(\mathrm{t}, J=7.4 \mathrm{~Hz}, 1 \mathrm{H}), 7.14-7.06(\mathrm{~m}$, $2 \mathrm{H}), 7.31(\mathrm{t}, J=7.2 \mathrm{~Hz}, 2 \mathrm{H}), 6.53(\mathrm{br}, 1 \mathrm{H}), 5.91(\mathrm{br}, 1 \mathrm{H}), 4.11(\mathrm{~s}, 3 \mathrm{H}), 3.57(\mathrm{~d}, J=6.4 \mathrm{~Hz}, 2 \mathrm{H}), 3.31(\mathrm{~d}$, $J=6.0 \mathrm{~Hz}, 2 \mathrm{H}), 0.73(\mathrm{~s}, 6 \mathrm{H}) .{ }^{13} \mathrm{C}-\mathrm{NMR}\left(101 \mathrm{MHz}, \mathrm{CDCl}_{3}\right): \delta=157.7,156.8,148.2,139.7,136.4,130.9$, 129.7 (2C), 127.3, 126.6, 124.0 (2C), 123.9, 123.7, 122.6, 120.6 (2C), 120.0, 117.9, 116.9, 116.4, 106.8, 55.9, 48.3, 38.4, 33.4, 24.1 (2C). HRMS (ESI): $m / z=484.1918\left[\mathrm{M}-\mathrm{H}^{-}\right.$, calcd. 485.1982.

1-(2-Methyl-1-(5-methyl-5H-indolo[2,3-b]quinolin-11-ylamino)propan-2-yl)-3-phenylurea 3d. Yellowish solid. Yield: $89 \%$. M.p. $175-177^{\circ} \mathrm{C} .{ }^{1} \mathrm{H}-\mathrm{NMR}\left(600 \mathrm{MHz}, \mathrm{CDCl}_{3}\right): \delta=8.88(\mathrm{br}, 1 \mathrm{H}), 8.31(\mathrm{~s}, 1 \mathrm{H}), 7.73(\mathrm{dd}$, $J=7.2,7.2 \mathrm{~Hz}, 2 \mathrm{H}), 7.51(\mathrm{t}, J=12 \mathrm{~Hz}, 4 \mathrm{H}), 7.37(\mathrm{~d}, J=8.4 \mathrm{~Hz}, 2 \mathrm{H}), 7.30(\mathrm{t}, J=7.4 \mathrm{~Hz}, 2 \mathrm{H}), 7.10(\mathrm{t}$, $J=7.2 \mathrm{~Hz}, 1 \mathrm{H}), 7.04(\mathrm{t}, J=7.0 \mathrm{~Hz}, 1 \mathrm{H}), 6.05(\mathrm{~s}, 1 \mathrm{H}), 4.07(\mathrm{~s}, 3 \mathrm{H}), 3.77(\mathrm{~s}, 2 \mathrm{H}), 1.17(\mathrm{~s}, 6 \mathrm{H}) .{ }^{13} \mathrm{C}-\mathrm{NMR}$ $\left(151 \mathrm{MHz}, \mathrm{CDCl}_{3}\right): \delta=157.2,156.9,151.9,148.7,139.8,136.5,130.9,129.6(2 \mathrm{C}), 129.5,127.1,126.2,124.3$, $123.3,122.3,119.9(2 \mathrm{C}), 119.7,117.4,117.0,116.3,106.4,60.1,54.6,33.4,26.3(2 \mathrm{C})$.

1-(2,2-Dimethyl-3-(5-methyl-5H-indolo[2,3-b]quinolin-11-ylamino)propyl)-3phenylthiourea 4a. Yellowish solid. Yield: $90 \%$. M.p. $172-174{ }^{\circ} \mathrm{C} .{ }^{1} \mathrm{H}-\mathrm{NMR}\left(600 \mathrm{MHz}, \mathrm{CDCl}_{3}\right): \delta=8.54(\mathrm{~d}, J=6 \mathrm{~Hz}, 1 \mathrm{H}), 7.86(\mathrm{~d}$, $J=6.0 \mathrm{~Hz}, 1 \mathrm{H}), 7.76(\mathrm{dd}, J=7.8,7.2 \mathrm{~Hz}, 2 \mathrm{H}), 7.66(\mathrm{~d}, J=7.8 \mathrm{~Hz}, 1 \mathrm{H}), 7.46(\mathrm{t}, J=6.0 \mathrm{~Hz}, 1 \mathrm{H}), 7.39(\mathrm{dd}$, $J=9,7.2 \mathrm{~Hz}, 3 \mathrm{H}), 7.32(\mathrm{~d}, J=6.0 \mathrm{~Hz}, 2 \mathrm{H}), 7.25-7.19(\mathrm{~m}, 1 \mathrm{H}), 6.89(\mathrm{~s}, 1 \mathrm{H}), 4.24(\mathrm{~s}, 3 \mathrm{H}), 3.79(\mathrm{~d}$, $J=6.0 \mathrm{~Hz}, 2 \mathrm{H}), 3.73(\mathrm{~d}, J=6.0 \mathrm{~Hz}, 2 \mathrm{H}), 1.96(\mathrm{br}, 1 \mathrm{H}), 0.71(\mathrm{~s}, 6 \mathrm{H}) .{ }^{13} \mathrm{C}-\mathrm{NMR}\left(151 \mathrm{MHz}, \mathrm{CDCl}_{3}\right)$ : $\delta=182.5,153.4,151.0,145.5,138.5,137.4,132.0,129.5$ (2C), 126.4, 126.24, 125.2 (2C), 125.0, 123.3, 123.1, 122.7, 121.1, 117.1, 115.4, 115.1, 103.5, 54.7, 51.8, 39.2, 34.6, 24.3 (2C). HRMS (ESI): $m / z=466.2170$ $[\mathrm{M}-\mathrm{H}]^{-}$, calcd. 467.2144 .

1-(1-(2-Chloro-5-methyl-5H-indolo[2,3-b]quinolin-11-ylamino)-2-methylpropan-2-yl)-3-phenylthiourea $\mathbf{4 b}$. Yellowish solid. Yield: $84 \%$. M.p. $147-149{ }^{\circ} \mathrm{C} .{ }^{1} \mathrm{H}-\mathrm{NMR}\left(600 \mathrm{MHz}, \mathrm{CDCl}_{3}\right): \delta=8.43(\mathrm{~s}, 1 \mathrm{H}), 8.09$ $(\mathrm{s}, 1 \mathrm{H}), 7.81(\mathrm{~d}, J=7.2 \mathrm{~Hz}, 1 \mathrm{H}), 7.68(\mathrm{~d}, J=8.4 \mathrm{~Hz}, 1 \mathrm{H}), 7.59-7.56(\mathrm{~m}, 1 \mathrm{H}), 7.48(\mathrm{~d}, J=12 \mathrm{~Hz}, 1 \mathrm{H}), 7.38$ $(\mathrm{t}, J=7.6 \mathrm{~Hz}, 1 \mathrm{H}), 7.33-7.29(\mathrm{~m}, 2 \mathrm{H}), 7.22(\mathrm{t}, J=7.5 \mathrm{~Hz}, 1 \mathrm{H}), 7.11(\mathrm{dd}, J=12.9,7.5 \mathrm{~Hz}, 3 \mathrm{H}), 6.13(\mathrm{~s}, 1 \mathrm{H})$, $5.88(\mathrm{~s}, 1 \mathrm{H}), 4.41(\mathrm{~d}, J=5.5 \mathrm{~Hz}, 2 \mathrm{H}), 4.06(\mathrm{~s}, 3 \mathrm{H}), 1.22(\mathrm{~s}, 6 \mathrm{H}) .{ }^{13} \mathrm{C}-\mathrm{NMR}\left(151 \mathrm{MHz}, \mathrm{CDCl}_{3}\right): \delta=180.6$, $156.5,152.8,147.9,136.8$ (d), 130.8, 130.4, 127.6, 126.8, 126.6, 125.5 (2C), 124.5, 124.4, 123.9, 121.7, 118.4, $117.6,117.1,116.6,109.1,60.8,58.7,55.5,33.3,26.7(2 \mathrm{C})$.

\subsection{Antitumor Screening Test}

Anti-proliferative assays in vitro were performed in the same manner as described in the literature [15,16,21,22]. The cell lines of human leukemia MV4-11, lung A549, and colon HCT 116 cancer were chosen as representing various tissue origins and BALB/3T3 normal murine fibroblasts to assess the cytotoxicity of tested compounds towards normal cells. The $\mathrm{IC}_{50}$ parameter (half-maximum inhibitory concentration) was calculated based on sulforhodamine B (HCT-116, A549, BALB/3T3) or 
MTT (MV4-11) assay results after $72 \mathrm{~h}$ of exposure $[15,16]$. Each compound in a given concentration was tested in triplicate in each experiment, which was repeated three to five times.

A lot of new chemicals undergo screening in various cell cultures but eventually do not achieve drug status due to severe side-effects. As Badisa et al. note: "Ideally, one of the criteria for a drug being good is that it should not exhibit any undesirable side-effects on normal cells" [23]. Therefore, to improve the search for new, not or significantly less toxic to normal mammalian cells compounds, the introduction of normal cells and a comparison of the cytotoxicity of tested compounds with cancer cells in early drug-screening protocols is valuable. Moreover, the BALB/3T3 fibroblasts used in our studies are recommended by various agencies introducing alternative methods for testing the toxicity of compounds. For example, these cells are used in some methods aiming to estimate starting doses for the oral acute systemic toxicity of compounds under the European Center of Validation of Alternative Methods (ECVAM) guidelines (OECD guidance document (GD) 129 published in 2010).

\section{Conclusions}

In conclusion, we have prepared a series of 11-substituted neocryptolepines with branched $\omega$-aminoalkylamino chains of different linker lengths between the two nitrogen atoms, and their antiproliferative activities were evaluated using the MV4-11 (human leukemia), A549 (human lung cancer), HCT116 (human colon cancer), and normal mouse fibroblast (BALB/3T3) cell lines. All synthesized compounds showed potent in vitro antiproliferative activity against the MV4-11 cell line over the 11-chloro substituted precursors. As a result of the diversification of the $\omega$-aminoalkylamino chains, we found the highest antiproliferative activity for 11-(3-amino2-hydroxy)propylamino-substituted compounds $\mathbf{2 h}$ and $\mathbf{2 k}$, showing mean $\mathrm{IC}_{50}$ values of $0.042 \mu \mathrm{M}$ and $0.057 \mu \mathrm{M}$ against MV4-11 cells, $0.197 \mu \mathrm{M}$ and $0.1988 \mu \mathrm{M}$ against A549 cells, and $0.138 \mu \mathrm{M}$ and $0.117 \mu \mathrm{M}$ against BALB/3T3 cells, respectively. The modification of $\omega$-aminoalkylamino chains to $\omega$-ureido and -thioureido derivatives was not the case for the improved activity. Further variations in substituents and their pattern may be necessary to obtain better activity.

Supplementary Materials: The NMR spectra of the compounds can be available online.

Acknowledgments: We are grateful to Okayama University for its support by Promotion of Graduate Course Students (to E.S.), and to the Advanced Science Research Center for the NMR experiments and the Elementary Analysis (E.S.) by M. Kosaka and M. Kobayashi. We are thankful to S. Nakashima, Okayama University, and X.-Q. Yu, Sichuan University, for the HRMS analyses, and to J. Futami for the UV analyses. This study was partially supported by the Adaptable and Seamless Technology Transfer Program from Japan Science and Technology Agency (JST), No. AS242Z02199Q.

Author Contributions: I.E.T.E.S., J.W., and T.I. designed the research; E.S., M.Ś., L.W., N.W., F.X., I.H., T.A.N., S.N., S.E.-G., S.S., and A.A.A.E.G. performed the research and analyzed the data; I. E.T.E.S., J.W., and T.I. wrote the paper. All authors read and approved the final manuscript.

Conflicts of Interest: The authors declare no conflict of interest.

\section{References}

1. Kumar, E.; Etukala, J.; Ablordeppey, S. Indolo[3,2-b]quinolines: Synthesis, biological evaluation and structure activity relationships. Mini-Rev. Med. Chem. 2008, 8, 538-554. [CrossRef] [PubMed]

2. Lavrado, J.; Moreira, R.; Paulo, A. Indoloquinolines as scaffolds for drug discovery. Curr. Med. Chem. 2010, 17, 2348-2370. [CrossRef] [PubMed]

3. Parvatkar, P.; Parameswaran, S.; Tilve, S. Isolation, biological activities and synthesis of indoloquinoline alkaloids: Cryptolepine, isocryptolepine and neocryptolepine. Curr. Org. Chem. 2011, 15, 1036-1057. [CrossRef]

4. Cimanga, K.; Bruyne, T.; Pieters, L.; Claeys, M.; Vlietinck, A. New alkaloids from Cryptolepis sanguinolenta. Tetrahedron Lett. 1996, 37, 1703-1706. [CrossRef]

5. Cimanga, K.; Bruyne, T.; Pieters, L.; Vlietinck, A.; Turger, C. In Vitro and in vivo antiplasmodial activity of cryptoleipine and related alkaloids from Cryptolepis sanguinolenta. J. Nat. Prod. 1997, 60, 688-691. [CrossRef] [PubMed] 
6. Paulo, A.; Gomes Elsa, T.; Steele, J.; Warhurst Dave, C.; Houghton Peter, J. Antiplasmodial activity of Cryptolepis sanguinolenta alkaloids from leaves and roots. Planta Med. 2000, 66, 30-34. [CrossRef] [PubMed]

7. Anash, C.; Otsyina, H.; Duwiejue, M.; Woode, E.; Aboagye, F.; Aning, K. Toxicological assessment of Cryptolepis sanguinolenta for possible use in veterinary medicine. J. Vet. Med. Anim. Health 2009, 1, 11-16.

8. Grellier, P.; Frappier, F.; Trigalo, F.; Ramiaramanana, L.; Millerioux, V.; Deharo, E.; Bodo, B.; Schrével, J.; Pousset, J.L. Antimalarial activity of alkaloids isolated from Cryptolepis sanguinolenta, cryptolepine and isocryptolepine. Phytother. Res. 1996, 10, 317-321. [CrossRef]

9. Kirby, G.; Paine, A.; Warhurst, D.; Noamese, B.; Phillipson, J. In vitro and in vivo antimalarial activity of cryptolepine, a plant-derived indoloquinoline. Phytother. Res. 1995, 9, 359-363. [CrossRef]

10. Wright, C.; Phillipson, J.; Awe, S.; Kirby, G.; Warhurst, D. Antimalarial activity of cryptolepine and some other anhydronium bases. Phytother. Res. 1996, 10, 361-363. [CrossRef]

11. Guittat, L.; Alberti, P.; Rosu, F.; Van Miert, S.; Thetiot, E.; Pieters, L.; Gabelica, V.; De Pauw, E.; Ottaviani, A.; Riou, J.; et al. Interactions of cryptolepine and neocryptolepine with unusual DNA structures. Biochimie 2003, 85, 535-547. [CrossRef]

12. Jonckers, T.; Van Miert, S.; Cimanga, K.; Bailly, C.; Colson, P.; De Pauw-Gillet, M.; Van Den Heuvel, H.; Claeys, M.; Lemière, F.; Esmans, E.; et al. Synthesis, cytotoxicity, and antiplasmodial and antitrypanosomal activity of new neocryptolepine derivatives. J. Med. Chem. 2002, 45, 3497-3508. [CrossRef] [PubMed]

13. Bailly, C.; Laine, W.; Baldeyrou, B.; De Pauw-Gillet, M.; Colson, P.; Houssier, C.; Cimanga, K.; Van Miert, S.; Vlietinck, A.; Pieters, L. DNA intercalation, topoisomerase II inhibition and cytotoxic activity of the plant alkaloid neocryptolepine. Anti-Cancer Drug Des. 2000, 15, 191-201.

14. Dassonneville, L.; Lansiaux, A.; Wattelet, A.; Wattez, N.; Mahieu, C.; Van Miert, S.; Pieters, L.; Bailly, C. Cytotoxicity and cell cycle effects of the plant alkaloids cryptolepine and neocryptolepine: Relation to drug-induced apoptosis. Eur. J. Pharmacol. 2000, 409, 9-18. [CrossRef]

15. Wang, L.; Switalska, M.; Mei, Z.; Lu, W.; Takahara, Y.; Feng, X.; El-Sayed, I.; Wietrzyk, J.; Inokuchi, T. Synthesis and in vitro antiproliferative activity of new 11-aminoalkylamino-substituted $5 \mathrm{H}$ - and $6 H$-indolo[2,3-b]quinolines; structure-activity relationships of neocryptolepines and 6-methyl congeners. Bioorg. Med. Chem. 2012, 20, 4820-4829. [CrossRef] [PubMed]

16. Lu, W.; Świtalska, M.; Wang, L.; Yonezawa, M.; El-Sayed, I.; Wietrzyk, J.; Inokuchi, T. In vitro antiproliferative activity of 11-aminoalkylamino-substituted $5 \mathrm{H}$-indolo[2,3- $b$ ]quinolines; improving activity of neocryptolepines by installation of ester substituent. Med. Chem. Res. 2013, 22, 4492-4504. [CrossRef]

17. El-Sayed, I.; Van der Veken, P.; Steert, K.; Dhooghe, L.; Hostyn, S.; Van Baelen, G.; Lemiere, G.; Maes, B.; Cos, P.; Maes, L.; et al. Synthesis and antiplasmodial activity of aminoalkylamino-substituted neocryptolepine derivatives. J. Med. Chem. 2009, 52, 2979-2988. [CrossRef] [PubMed]

18. Wang, L.; Lu, W.; Odawara, T.; Misumi, R.; Mei, Z.; Peng, W.; El-Sayed, I.; Inokuchi, T. Improved synthesis and reaction of 11-chloroneocryptolepines, strategic scaffold for antimalarial agent, and their 6-methyl congener from indolo-3-carboxylate. J. Heterocycl. Chem. 2014, 51, 1106-1114. [CrossRef]

19. Mei, Z.; Wang, L.; Lu, W.; Pang, C.; Maeda, T.; Peng, W.; Kaiser, M.; El-Sayed, I.; Inokuchi, T. Synthesis and in vitro antimalarial testing of neocryptolepines: SAR study for improved activity by introduction and modification of side chains at C2 and C11 on 5H-indolo[2,3-b]quinolones. J. Med. Chem. 2013, 56, 1431-1442. [CrossRef] [PubMed]

20. Okada, M.; Mei, Z.-W.; Hossain, M.I.; Wang, L.; Tominaga, T.; Takebayashi, T.; Murakami, M.; Yasuda, M.; Shigehiro, T.; Kasai, T.; et al. Synthesis, in-vitro cancer cell growth inhibition evaluation of 11-modified indolo[2,3-b] quinolines and their COMPARE analyses. Med. Chem. Res. 2016, 25, 879-914. [CrossRef]

21. Rubinstein, L.; Shoemaker, P.; Paull, K.; Simon, M.; Tosini, S.; Skehan, P.; Scudiero, D.; Monks, A.; Boyd, M. Comparison of in vitro anticancer-drug-screening data generated with a tetrazolium assay versus a protein assay against a diverse panel of human tumor cell lines. J. Natl. Cancer Inst. 1990, 82, 1113-1118. [CrossRef] [PubMed]

22. Purcell, M.; Neault, J.; Tajmir-Riahi, H. Interaction of taxol with human serum albumain. Biochim. Biophys. Acta Protein Struct. Mol. Enzymol. 2000, 1478, 61-68. [CrossRef] 
23. Badisa, R.; Darling-Reed, S.; Joseph, P.; Cooperwood, J.; Latinwo, L.; Goodman, C. Selective cytotoxic activities of two novel synthetic drugs on human breast carcinoma MCF-7 cells. Anticancer Res. 2009, 29, 2993-2996. [PubMed]

Sample Availability: Samples of the compound 2 are available from the authors.

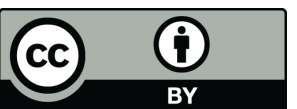

(C) 2017 by the authors. Licensee MDPI, Basel, Switzerland. This article is an open access article distributed under the terms and conditions of the Creative Commons Attribution (CC BY) license (http://creativecommons.org/licenses/by/4.0/). 